\section{BS18 FUNCTIONAL AND MOLECULAR ANALYSIS OF ABERRANT EXPRESSION OF MICRORNA-133A IN ENDOTHELIAL CELLS DURING CARDIOVASCULAR DISEASE}

${ }^{1}$ Suhail Ahmed*, ${ }^{2}$ Satishkumar Kurusamy, ${ }^{3}$ Angel L Armesilla, ${ }^{4}$ James Cotton. ${ }^{1}$ RIHS, FSE, University of Wolverhampton; ${ }^{2}$ RIHS, FSE, University of Wolverhampton; ${ }^{3}$ RIHS, FSE, University of Wolverhampton; ${ }^{4}$ Department of Cardiology, Heart and Lung Centre, New Cross Hospital, Wolverhampton, UK

\subsection{6/heartjnl-2019-BCS.181}

Introduction MicroRNA (miRNA) molecules are a class of small non-coding RNA molecules ( -22 nucleotides), which target the 3' untranslated region (3' UTR) of mRNA and either induce mRNA degradation or suppress protein translation. Emerging evidence indicates miRNA molecules play a key role in the regulation of cardiovascular pathophysiology. miR-133a is mainly expressed in cardiomyocytes and skeletal muscle cells. In endothelial cells miR-133a is expressed at very low levels in physiological conditions however, increased expression of this microRNA in the endothelium has been strongly associated to cardiovascular disease. Although aberrant expression of miR-133a has been linked to endothelial dysfunction, the molecular and cellular mechanisms deregulated in endothelial cells by high expression of miR-133a remain largely unknown.

Methods Here, we have evaluated the consequences of aberrant expression of miR-133a in endothelial cells by transfecting primary Human Umbilical Vein Endothelial cells (HUVEC) with a double-stranded, miRNA-like mimic of miR-133a. This strategy simulates the situation observed in pathological conditions. A scramble miRNA-like mimic was used as negative control. The effect of "miR-133a mimic" in endothelial cell migration and tubular morphogenesis has been determined by performing wound-healing migration and matrigel assays. Changes in the expression of angiogenic genes caused by overexpression of miR-133a have been investigated by qPCR.

Results We show here that ectopic expression of miR-133a in endothelial cells robustly attenuates endothelial cell migration and VEGF-induced angiogenesis. As a first step to elucidate the molecular mechanisms underlying this inhibitory effect of miR-133a, we have screened gene arrays to identify changes in the expression of genes involved in cell motility and angiogenic signalling, and validated potential changes in gene expression by qPCR. Our results show that transfection of "miR-133a mimic" into primary endothelial cells strongly downregulates the expression of genes implicated in cell motility (such as PLAUR and MSN) and angiogenic signalling (such as CD44 and ID1).

Conclusion These data indicate that enhanced expression of miR-133a in endothelial cells impairs pro-angiogenic cellular processes by altering the expression of specific, target genes. Our results suggest that blockage of miR-133a function in endothelial cells might have important therapeutic applications to treat patients suffering from cardiovascular pathologies that occur with excessive angiogenesis.

Conflict of interest None

\section{BS19 BH4 SUPPLEMENTATION AS A NEW TREATMENT FOR DIABETIC CARDIOMYOPATHY}

${ }^{1}$ Ricardo Carnicer*, ${ }^{2}$ Klemen Ziberna, ${ }^{3}$ Ritu Arya, ${ }^{4}$ Keith Channon, ${ }^{5}$ Barbara Casadei. ${ }^{1}$ University of Oxford; ${ }^{2}$ NHS; ${ }^{3}$ University of Oxford; ${ }^{4}$ NIHR University of Oxford BRC and Oxford University Hospitals NHS Foundation Trust; ${ }^{5}$ university of Oxford

\subsection{6/heartjnl-2019-BCS.182}

Background and Aims: BH4 is successfully used in the clinic for inherited $\mathrm{BH} 4$ deficiency and $\mathrm{BH} 4$-responsive phenylketonuria. In recent years, BH4 supplementation has also drawn attention as a therapy for various nitric oxide synthase (NOS)related cardiovascular pathologies. By genetic intervention, we have been able to increase cardiac intracellular $\mathrm{BH} 4$ levels, modify cardiac metabolism and prevent heart dysfunction in a murine model of diabetic cardiomyopathy. The aim of this study was to assess the efficacy of an oral $\mathrm{BH} 4$ preparation in our animal model before translating the treatment into diabetic patients. In particular, we tested whether $\mathrm{BH} 4$ oral supplementation would be sufficient to increase $\mathrm{BH} 4 / \mathrm{NO}$ levels in cardiac tissue and if this would protect the diabetic heart.

Results and Methods: Diabetes was induced by streptozotocin injections over 5 consecutive days in WT mice. After 12 weeks of diabetes, mice were fed with either placebo or $\mathrm{BH} 4$ diet $(200 \mathrm{mg} / \mathrm{kg} /$ day $)$ for an additional 6 weeks. At the end of this period, the group of diabetic mice treated with $\mathrm{BH} 4$ showed a significant increase of this biopterin in the heart $(9.4 \pm 1.3 \mathrm{pmol} / \mathrm{mg}$ protein vs $5.8 \pm 0.8 \mathrm{pmol} / \mathrm{mg}$ in non-supplemented WT. $\mathrm{P}=0.034$. $\mathrm{N}=8$ hearts per group), as well as an increase in the activity of NOS $(0.6 \pm 0.11$ vs $0.2 \pm 0.05$ $\%$ citrulline conversion. $\mathrm{P}=0.009$ ).

WT diabetic mice showed impaired diastolic function as indicated by tissue Doppler analysis (Lower E'/A' ratio, $\mathrm{P}<0.001$ and a higher $\mathrm{E} / \mathrm{E}^{\prime}$ ratio, $\mathrm{P}<0.01$. $\mathrm{N}=16$ mice per group), as well as a reduction in the ejection fraction $(\mathrm{P}<0.01)$ independent of any decrease in $\mathrm{NO}$ or $\mathrm{BH} 4$ bioavailability. In contrast, diabetic treated with $\mathrm{BH} 4$ displayed preserved cardiac function. Similarly, isolated cardiomyocytes from $\mathrm{BH} 4-\mathrm{fed}$ diabetic mice showed preserved time to $50 \%$ relaxation and decay of intracellular calcium transients $(n=53-$ 71 cells from 5-6 hearts per group) indicating that the cardioprotective effect exerted by $\mathrm{BH} 4$ was intrinsic to the myocardium.

Conclusion Oral $\mathrm{BH} 4$ was sufficient to increase the level of this biopterin and $\mathrm{NO}$ availability in cardiac tissue. As a result, cardiac function was preserved in a mouse model of diabetic cardiomyopathy. These results have prompted us to test the effects of $\mathrm{BH} 4$ on cardiac metabolism and function in diabetic patients.

Conflict of interest None

\section{BS20 COMPARISON OF VARIOUS ANTICOAGULANTS ON CLOT STRUCTURE IN ATRIAL FIBRILLATION}

${ }^{1}$ Ahsan Khan ${ }^{*},{ }^{2}$ Eduard Shantsila, ${ }^{2}$ Y.C. Lau, ${ }^{3}$ Lewis Hardy, ${ }^{3}$ Helen Philippou, ${ }^{1}$ Gregory Lip. ${ }^{1}$ Liverpool Heart \& Chest Hospital NHS Foundation Trust; ${ }^{2}$ University of Birmingham; ${ }^{3}$ University of Leeds

10.1136/heartjnl-2019-BCS.183

Introduction Atrial fibrillation (AF) is associated with an increased risk of stroke and thromboembolism. Despite best 